V. Rakic - W. A. Davis - S. A. P. Chubb •

F. M. A. Islam - R. L. Prince - T. M. E. Davis

\title{
Bone mineral density and its determinants in diabetes: the Fremantle Diabetes Study
}

Received: 24 October 2005 / Accepted: 30 November 2005 / Published online: 4 March 2006

C) Springer-Verlag 2006

\begin{abstract}
Aims/hypothesis: We assessed the effects of type 1 and type 2 diabetes on bone density and metabolism. Materials and methods: We analysed bone mineral density (BMD) measured at the hip, spine and forearm using dual energy X-ray absorptiometry in 34 patients with type 1 and 194 patients with type 2 diabetes. Patients were from the community-based Fremantle Diabetes Study, and findings for them were compared with those from normal age- and sex-matched control subjects from the local community. Biochemical and hormonal markers of bone metabolism were measured in a subset of 70 patients. Results: After adjusting for age and BMI, there was a lower BMD at total hip $(p<0.001)$ and femoral neck $(p=0.012)$ in type 1 men vs control subjects, but type 1 women and matched controls had similar BMD at each site. There was a higher BMD at total hip $(p=0.006)$, femoral neck $(p=0.026)$ and forearm $(p<0.001)$ in type 2 women vs control subjects, but diabetes status was not associated with BMD in type 2 men after adjustment for age and BMI. Serum oestradiol, BMI, C-terminal telopeptide of collagen type 1 and male sex were consistently and independently associated with BMD at forearm, hip and femoral neck and explained 61, 55 and
\end{abstract}

\footnotetext{
V. Rakic · W. A. Davis · T. M. E. Davis $(\triangle)$

University of Western Australia, School of Medicine

and Pharmacology, Fremantle Hospital,

P.O. Box 480, Fremantle, WA 6959, Australia

e-mail: tdavis@cyllene.uwa.edu.au

Tel.: +61-8-94313229

Fax: +61-8-94312977

S. A. P. Chubb

Biochemistry Department, Fremantle Hospital,

Fremantle, WA, Australia

F. M. A. Islam · R. L. Prince

Department of Endocrinology and Diabetes,

Sir Charles Gairdner Hospital,

Nedlands, WA, Australia

R. L. Prince

University of Western Australia, School of Medicine and Pharmacology, Sir Charles Gairdner Hospital,

Nedlands, WA, Australia
}

$50 \%$ of the total variance in $\mathrm{BMD}$, respectively, at these sites. Spine BMD was independently associated with BMI and $\ln$ (oestradiol). Conclusions/interpretation: Men with type 1 diabetes may be at increased risk of osteoporosis, while type 2 women appear to be protected even after adjusting for BMI. Low serum oestradiol concentrations may predispose to diabetes-associated osteoporosis regardless of sex.

Keywords Bone mineral density $\cdot$ Bone turnover markers - Diabetes mellitus - Dual energy X-ray absorptiometry $\cdot$ Sex hormones

Abbreviations 25OHD: 25-hydroxyvitamin D - ALP: alkaline phosphatase - BMD: bone mineral density $\cdot$ CTx: carboxyl-terminal type I collagen telopeptide $\cdot$ E2: oestradiol - FDS: Fremantle Diabetes Study · FSH: folliclestimulating hormone $\cdot$ HRT: hormone replacement therapy - LH: luteinising hormone - PTH: parathyroid hormone $\cdot$ SHBG: sex hormone-binding globulin

\section{Introduction}

The relationship between diabetes and osteoporosis is complex. A history of diabetes, diabetes duration and chronic complications are associated with an increased risk of osteoporotic fracture $[1,2]$, while the fracture site may also be influenced by whether or not the patient has diabetes [3]. The association between low bone mineral density (BMD) and diabetes has most consistently been observed in type 1 patients $[4,5]$, with increased bone mineral loss attributed to early age at diagnosis, long duration, prolonged poor glycaemic control and high insulin doses [6-9]. In type 2 diabetes, the relationship is less clear, with reports of increased [10], decreased [11] or unchanged [12] bone mass and BMD, probably reflecting the heterogeneity of the disease and/or the different study methodologies used.

Several mechanisms may contribute to type 1 diabetesassociated osteopenia, including impaired vitamin D-para- 
thyroid hormone $(\mathrm{PTH})$ regulation $[9,13]$ and altered bone mineral metabolism [14]. Increased bone resorption and decreased formation have been observed in young poorly controlled patients with type 1 diabetes of long duration $[15,16]$. By contrast, low bone turnover has been reported in type 2 diabetes, particularly in insulin-treated patients [17]. Although sex steroids play a significant role in skeletal metabolism in the general population [18-20], there are few equivalent data from diabetic patients.

We therefore investigated the relationship between BMD and diabetes in well-characterised type 1 and type 2 patients from a representative community-based cohort who were matched with healthy non-diabetic controls from the local population. We also examined the role of bone turnover markers and sex steroids in modulating BMD in diabetes.

\section{Subjects, materials and methods}

\section{Subjects}

We studied 231 patients from the Fremantle Diabetes Study (FDS), a community-based prospective observational study conducted in a postcode-defined population of 120,097 surrounding Fremantle in Western Australia. The FDS protocol and present sub-study were approved by the Fremantle Hospital Human Rights Committee and informed consent was obtained from each patient before participation in both. Between 1993 and 1996, we identified 2,258 eligible patients and recruited 1,426 (63\%) for annual assessments, including 127 with type 1 diabetes, 1,294 with type 2 and five with secondary diabetes. Details of identification and recruitment, sample characteristics, diabetes classification by clinical/laboratory criteria and particulars of non-recruited patients have been described elsewhere [21]. The present subset of FDS patients was a convenience sample of those without a selfreported history of osteoporosis who were not taking medication for the disease and who attended an annual assessment during 2000 and 2001. All the present patients were matched individually by age and sex with nondiabetic individuals recruited from the local healthy population whose BMD had been measured for determination of reference ranges [22].

\section{Clinical assessment}

Data relating to diabetes, comorbidities and other relevant variables (including exercise levels and tobacco and alcohol consumption) from the FDS annual assessment prior to entry into the present sub-study (an average of 6 months beforehand) were available for each patient. At the present sub-study assessment, weight and height were remeasured. All patients completed a supplementary questionnaire which contained questions relating to (1) conditions predisposing to osteoporosis (family history, hepatic and renal disease, thyroid and parathyroid disease, inflammatory conditions including rheumatoid arthritis, malabsorption, glucocorticoid use, menopausal status and use of hormone replacement therapy [HRT]); (2) details of past fractures; (3) prior use of any treatment for osteoporosis or any medication influencing bone metabolism; and (4) details of previous bone density measurements. Additional data on fractures were obtained from hospitalisations recorded in the Western Australian Data Linkage System [23].

Nephropathy was taken as a urinary albumin:creatinine ratio $>3.0 \mathrm{mg} / \mathrm{mmol}$ on a first-morning urine sample. Peripheral sensory neuropathy was defined as a score of $>2 / 8$ on the clinical portion of the Michigan Neuropathy Screening Instrument [24]. A subject was considered as having retinopathy if any grade of retinopathy, including maculopathy, was detected by direct and/or indirect ophthalmoscopy in one or both eyes and/or on more detailed assessment by an ophthalmologist. Self-reported stroke and transient ischaemic attack were amalgamated with prior hospitalisations to define baseline cerebrovascular disease status [23]. Patients were classified as having CHD if there was a self-reported history of, or hospitalisation for, myocardial infarction, angina, coronary artery bypass grafting, angioplasty, and/or definite myocardial infarction on a Minnesota-coded electrocardiogram [25].

\section{Bone densitometry}

Each patient attended for measurement of BMD at hip, spine (L1-L4) and forearm using dual-energy X-ray absorptiometry (QDR-4500W; Hologic, Bedford, MA, USA) from which T-scores were generated for diabetic patients. For the control subjects for whom T-scores had not been generated, the formula (BMD-young normal mean)/SD was used. Osteoporosis was defined as a T-score at the hip of less than -2.5 [26] and osteopenia as a T-score of $-1.0 \mathrm{SD}$ to $-2.5 \mathrm{SD}$. The CV of T-scores was $1.1 \%$ at the spine and $1.0-1.2 \%$ at the hip [27]. Valid BMD data for two or more sites were obtained for 229 patients $(99.1 \%$ of the sample), of whom 34 had type 1 and 194 type 2 diabetes.

\section{Measurement of biochemical and hormonal variables}

Stored sera from 33 of the 34 type 1 patients were available for measurement of markers and determinants of bone turnover comprising serum calcium, phosphate, albumin, osteocalcin, carboxyl-terminal type I collagen telopeptide (CTx), alkaline phosphatase (ALP), 25-hydroxyvitamin D (25OHD), total testosterone, estradiol (E2), sex hormonebinding globulin (SHBG), luteinising hormone (LH), follicle-stimulating hormone (FSH) and plasma PTH. In addition, sera from a subgroup of 37 of the 194 type 2 diabetic patients, matched for sex and as closely as possible for serum creatinine with the type 1 patients, were also retained for the same range of assays.

Serum calcium, phosphate, albumin and ALP were measured spectrophotometrically (Cobas Integra 800; 
Roche Diagnostics, Castle Hill, NSW, Australia). Betweenday $\mathrm{CV}$ was $1.8 \%$ at $2.0 \mathrm{mmol} / \mathrm{l}$ calcium, $3.8 \%$ at $0.7 \mathrm{mmol} / 1$ phosphate and $7.8 \%$ at $234 \mathrm{U} / 1$ ALP. Plasma $\mathrm{PTH}$, serum beta-CTx and osteocalcin were measured using electrochemiluminescent immunoassays (Elecsys 2010 analyser; Roche Diagnostics) with between-day CVs of $4.7 \%$ at $3.6 \mathrm{pmol} / \mathrm{l}$ for PTH, $4.3 \%$ at $0.39 \mathrm{mg} / \mathrm{ml}$ $\mathrm{CTx}$ and $1.8 \%$ at $68 \mathrm{ng} / \mathrm{ml}$ osteocalcin. Total serum testosterone was measured by a coated-tube RIA and E2 by a double-antibody RIA (DPC-BioMediq, Doncaster, VIC, Australia). Between-day imprecision was $8.5 \%$ at $14 \mathrm{nmol} / 1$ testosterone, $11.9 \%$ at $44 \mathrm{pmol} / \mathrm{l}$ E2 and $10.0 \%$ at $121 \mathrm{pmol} / \mathrm{l} \mathrm{E} 2$ with the lower limit of E2 detection at $10 \mathrm{pmol} / \mathrm{l}$. SHBG, LH and FSH were measured by chemiluminescent immunoassay (Immulite 2000 analyser; DPC-BioMediq). Between-day imprecision was $5.5 \%$ at 16 $\mathrm{U} / 1 \mathrm{LH}, 5.5 \%$ at $14.4 \mathrm{U} / \mathrm{l} \mathrm{FSH}$ and $9.4 \%$ at $74 \mathrm{nmol} / 1$ SHBG. 25OHD was measured by RIA (Diasorin; Vital Diagnostics, Cleveland, QLD, Australia). Between-day imprecision was $8.4 \%$ at $43 \mathrm{nmol} / \mathrm{l}$. Free testosterone was calculated for patients with E2 $<150 \mathrm{pmol} / 1$ [28].

\section{Statistical analysis}

Statistical analyses were performed using SPSS for Windows (version 11; SPSS, Chicago, IL, USA) and SAS for Windows (version 8.02; SAS Institute, Cary, NC, USA). Data are presented as proportions, means $\pm \mathrm{SD}$, geometric mean (SD range) or, in the case of variables not conforming to a normal or log-normal distribution, median [interquartile range]. Two unrelated groups were compared by Fisher's exact test, Student's $t$-test or Mann-Whitney $U$ test. Two related normally distributed groups were compared using the paired $t$-test. Since age and BMI are known risk factors for low BMD [29, 30], general linear modelling was used to compare BMD between type 1 and type 2 diabetes after adjusting for these variables. Likewise, after adjusting for age and BMI, the effect of diabetic status on BMD was examined using a Generalised Estimating Equations procedure that recognised matching. The Wald statistic for type 3 Generalised Estimating Equation analysis was reported. Multiple linear regression using forward conditional modelling with entry $p<0.05$ and removal $p>0.10$ was used to determine independent associates of BMD. A significance level of $p<0.05$ was used throughout.

\section{Results}

Subject characteristics

We studied 23 men and 11 women with type 1 diabetes, and 108 men and 86 women with type 2 diabetes. Details of these patients and the matched controls are shown in Tables 1 and 2. The type 1 males were younger, had lower BMI, higher HDL-cholesterol, and longer disease duration than the type 2 males, but glycaemic control, BP, serum creatinine and neuropathy were not significantly different by diabetes type. The 11 type 1 women were significantly younger, had lower BMI, systolic BP and serum triglycerides, but higher $\mathrm{HbA}_{1 \mathrm{c}}$, and were less likely to have neuropathy than the 86 with type 2 diabetes, but disease duration and serum creatinine were not significantly different. A higher proportion of women with type 2 diabetes were postmenopausal with approximately 1 in 10 on HRT.

Table 1 Demographic, physical and biochemical variables in patients with type 1 and type 2 diabetes

\begin{tabular}{|c|c|c|c|c|c|c|}
\hline & \multicolumn{3}{|l|}{ Females } & \multicolumn{3}{|l|}{ Males } \\
\hline & Type 1 & Type 2 & $p$ value & Type 1 & Type 2 & $p$ value \\
\hline Number & 11 & 86 & - & 23 & 108 & - \\
\hline Age (years) & $43.5 \pm 9.7$ & $65.5 \pm 9.6$ & $<0.001$ & $54.4 \pm 14.3$ & $66.0 \pm 9.1$ & 0.001 \\
\hline Diabetes duration (years) & $9.7[7.1-27.0]$ & $9.1[7.1-11.9]$ & 0.74 & $18.8[11.5-30.1]$ & $8.7[6.9-12.7]$ & $<0.001$ \\
\hline BMI $\left(\mathrm{kg} / \mathrm{m}^{2}\right)$ & $26.1 \pm 5.2$ & $31.9 \pm 6.1$ & 0.004 & $25.9 \pm 3.2$ & $29.0 \pm 4.4$ & 0.002 \\
\hline Fasting plasma glucose $(\mathrm{mmol} / \mathrm{l})$ & $8.3[4.8-16.0]$ & $8.6[7.4-10.9]$ & 0.62 & $8.7[7.1-12.5]$ & $8.8[7.4-10.3]$ & 0.76 \\
\hline $\mathrm{HbA}_{1 \mathrm{c}}(\%)$ & $8.2[7.9-8.9]$ & $7.4[6.5-8.4]$ & 0.043 & $7.8[6.7-8.7]$ & $7.4[6.5-8.2]$ & 0.17 \\
\hline Systolic BP (mmHg) & $111 \pm 14$ & $137 \pm 19$ & $<0.001$ & $146 \pm 27$ & $144 \pm 20$ & 0.71 \\
\hline Diastolic BP (mmHg) & $66 \pm 11$ & $68 \pm 11$ & 0.56 & $75 \pm 7$ & $76 \pm 10$ & 0.76 \\
\hline Total serum cholesterol (mmol/l) & $5.4 \pm 0.9$ & $5.2 \pm 1.0$ & 0.50 & $5.0 \pm 1.4$ & $5.1 \pm 1.2$ & 0.77 \\
\hline HDL-cholesterol (mmol/l) & $1.52 \pm 0.44$ & $1.33 \pm 0.70$ & 0.39 & $1.51 \pm 0.44$ & $1.21 \pm 0.39$ & 0.002 \\
\hline Serum triglycerides $(\mathrm{mmol} / \mathrm{l})$ & $1.0[0.6-1.8]$ & $1.7[1.0-2.8]$ & 0.001 & $1.1[0.5-2.2]$ & $1.5[0.9-2.6]$ & 0.009 \\
\hline Serum creatinine $(\mu \mathrm{mol} / \mathrm{l})$ & $83 \pm 13$ & $75 \pm 24$ & 0.28 & $122 \pm 67$ & $94 \pm 33$ & 0.06 \\
\hline Peripheral neuropathy (\%) & 18.2 & 57.7 & 0.022 & 47.6 & 52.4 & 0.81 \\
\hline Postmenopausal (\%) & 33.3 & 90.8 & $<0.001$ & - & - & - \\
\hline Hormone replacement therapy (\%) & 0 & 10.5 & 0.59 & - & - & - \\
\hline
\end{tabular}

Data are proportions, means $\pm \mathrm{SD}$, geometric mean (SD range) or median [interquartile range] 
Table 2 Bone mineral density and T-score comparisons for cases and age- and sex-matched controls by type of diabetes

Female cases Female controls Unadjusted Adjusted $^{\mathrm{a}}$ Male cases Male controls Unadjusted Adjusted $^{\mathrm{a}}$ $p$ value $\quad p$ value $p$ value $\quad p$ value

\begin{tabular}{|c|c|c|c|c|c|c|c|c|}
\hline \multicolumn{9}{|l|}{ Type 1 diabetes } \\
\hline Number & 11 & 11 & - & - & 23 & 23 & - & - \\
\hline Age & $43.5 \pm 9.7$ & $43.1 \pm 8.8$ & 0.93 & - & $54.4 \pm 14.3$ & $54.7 \pm 14.7$ & 0.94 & - \\
\hline BMI $\left(\mathrm{kg} / \mathrm{m}^{2}\right)$ & $26.1 \pm 5.2$ & $24.9 \pm 5.0$ & 0.53 & - & $25.9 \pm 3.2$ & $27.3 \pm 2.4$ & 0.10 & - \\
\hline Hip $\left(\mathrm{g} / \mathrm{cm}^{2}\right)$ & $0.885 \pm 0.123$ & $0.949 \pm 0.128$ & 0.19 & 0.06 & $0.947 \pm 0.178$ & $1.087 \pm 0.178$ & 0.002 & $<0.001$ \\
\hline Hip T-score & $-0.753 \pm 1.025$ & $-0.218 \pm 1.063$ & 0.24 & 0.06 & $-0.964 \pm 1.371$ & $0.119 \pm 1.370$ & 0.010 & $<0.001$ \\
\hline Femoral neck $\left(\mathrm{g} / \mathrm{cm}^{2}\right)$ & $0.789 \pm 0.098$ & $0.819 \pm 0.127$ & 0.55 & 0.34 & $0.775 \pm 0.143$ & $0.877 \pm 0.153$ & 0.011 & 0.012 \\
\hline Femoral neck T-score & $-1.064 \pm 0.982$ & $-0.765 \pm 1.272$ & 0.54 & 0.34 & $-1.857 \pm 1.303$ & $-0.926 \pm 1.395$ & 0.024 & 0.012 \\
\hline Spine $\left(\mathrm{g} / \mathrm{cm}^{2}\right)$ & $1.031 \pm 0.114$ & $1.051 \pm 0.159$ & 0.72 & 0.61 & $1.011 \pm 0.196$ & $1.095 \pm 0.151$ & 0.051 & 0.08 \\
\hline Spine T-score & $-0.147 \pm 1.038$ & $0.032 \pm 1.449$ & 0.74 & 0.61 & $-0.726 \pm 1.783$ & $0.035 \pm 1.373$ & 0.11 & 0.08 \\
\hline Forearm $\left(\mathrm{g} / \mathrm{cm}^{2}\right)$ & $0.551 \pm 0.039$ & $0.557 \pm 0.064$ & 0.78 & 0.58 & $0.622 \pm 0.073$ & $0.639 \pm 0.061$ & 0.23 & 0.30 \\
\hline Forearm T-score & $-0.259 \pm 0.756$ & $-0.136 \pm 1.245$ & 0.78 & 0.58 & $-1.059 \pm 1.359$ & $-0.738 \pm 1.122$ & 0.39 & 0.30 \\
\hline \multicolumn{9}{|l|}{ Type 2 diabetes } \\
\hline Number & 86 & 86 & - & - & 108 & 108 & - & - \\
\hline Age & $65.5 \pm 9.6$ & $64.8 \pm 11.1$ & 0.66 & - & $66.0 \pm 9.1$ & $66.3 \pm 9.6$ & 0.80 & - \\
\hline BMI $\left(\mathrm{kg} / \mathrm{m}^{2}\right)$ & $31.9 \pm 6.1$ & $25.1 \pm 3.5$ & $<0.001$ & - & $29.0 \pm 4.3$ & $25.9 \pm 3.0$ & $<0.001$ & - \\
\hline $\operatorname{Hip}\left(\mathrm{g} / \mathrm{cm}^{2}\right)$ & $0.993 \pm 0.173$ & $0.848 \pm 0.118$ & $<0.001$ & 0.006 & $1.060 \pm 0.156$ & $1.013 \pm 0.158$ & 0.038 & 0.88 \\
\hline Hip T-score & $0.148 \pm 1.440$ & $-1.056 \pm 0.983$ & $<0.001$ & 0.006 & $-0.091 \pm 1.197$ & $-0.456 \pm 1.218$ & 0.029 & 0.88 \\
\hline Femoral neck $\left(\mathrm{g} / \mathrm{cm}^{2}\right)$ & $0.808 \pm 0.153$ & $0.722 \pm 0.103$ & $<0.001$ & 0.026 & $0.851 \pm 0.128$ & $0.802 \pm 0.129$ & 0.010 & 0.34 \\
\hline Femoral neck T-score & $-0.870 \pm 1.527$ & $-1.726 \pm 1.035$ & $<0.001$ & 0.026 & $-1.167 \pm 1.168$ & $-1.608 \pm 1.177$ & 0.007 & 0.34 \\
\hline Spine $\left(\mathrm{g} / \mathrm{cm}^{2}\right)$ & $1.031 \pm 0.171$ & $0.948 \pm 0.152$ & 0.001 & 0.59 & $1.117 \pm 0.176$ & $1.102 \pm 0.191$ & 0.55 & 0.72 \\
\hline Spine T-score & $-0.144 \pm 1.553$ & $-0.897 \pm 1.379$ & 0.001 & 0.59 & $0.239 \pm 1.600$ & $0.101 \pm 1.737$ & 0.54 & 0.72 \\
\hline Forearm $\left(\mathrm{g} / \mathrm{cm}^{2}\right)$ & $0.540 \pm 0.066$ & $0.481 \pm 0.068$ & $<0.001$ & $<0.001$ & $0.641 \pm 0.062$ & $0.627 \pm 0.063$ & 0.09 & 0.54 \\
\hline Forearm T-score & $-0.462 \pm 1.291$ & $-1.635 \pm 1.327$ & $<0.001$ & $<0.001$ & $-0.702 \pm 1.143$ & $-0.963 \pm 1.163$ & 0.10 & 0.54 \\
\hline
\end{tabular}

Data are means \pm SD

${ }^{\mathrm{a}}$ Adjusted for age and BMI using General Estimating Equation procedures that recognised matching

BMD in type 1 diabetes

BMD and T-score data are summarised in Table 2 and Fig. 1. Type 1 men had significantly lower BMD at hip and femoral neck compared with controls but total spine and forearm

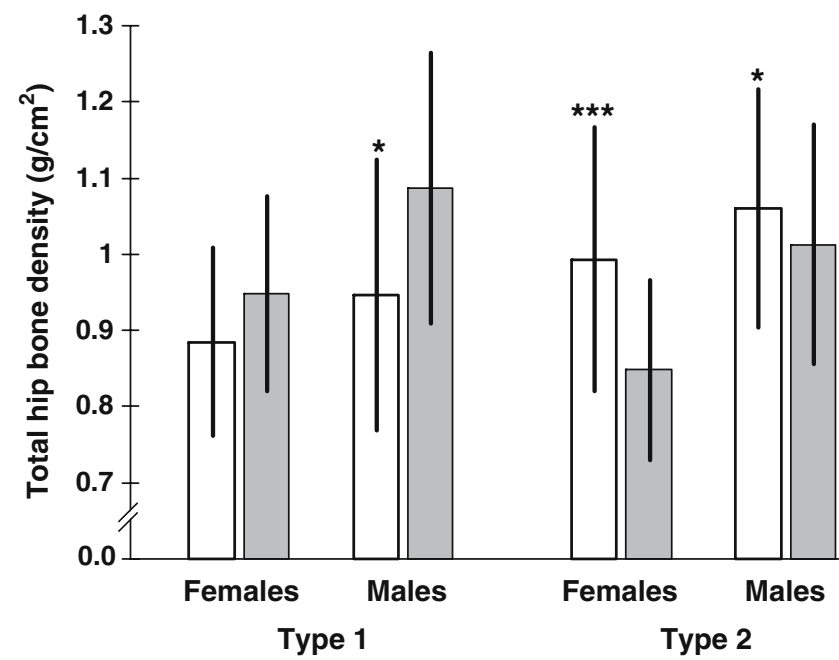

Fig. 1 Total hip bone mineral density (unadjusted) for cases with type 1 and type 2 diabetes (open bars) and age- and sex-matched controls (filled bars). ${ }^{*} p<0.05 ; * * * p<0.001$
BMD were not significantly different. After adjusting for age and BMI, the lower BMD at femoral neck and hip in type 1 men vs controls persisted. There were no significant differences in BMD or T-score at any site in type 1 vs control women, although the observed differences were in the same direction as for the men.

Categories of bone density for the type 1 patients are shown in Table 3. A significantly higher proportion of type 1 diabetic men had osteopenia/osteoporosis at each site than controls. However, this was statistically significant only at the hip and femoral neck, and only at the latter site after adjustment for age and BMI. The distribution of BMD categories in women with type 1 diabetes did not differ from that in matched control subjects.

BMD in type 2 diabetes

BMD and T-score data for the type 2 patients and controls are summarised in Table 2 and Fig. 1. In contrast to the type 1 patients, both the men and women with type 2 diabetes had higher BMD and T-score at the hip (total and femoral neck) than the controls. In addition, in women with type 2 diabetes, spine and forearm BMD and T-score were higher than for control females. The differences in the women persisted at the femoral neck, total hip and forearm, but not 
Table 3 Categories of bone density (normal/osteopenia/osteoporosis) for diabetic cases and age- and sex-matched controls

$$
\text { Female cases (\%) } \quad \text { Female controls (\%) } \quad p \text { value }^{\mathrm{a}} \text { Adjusted } \text { Male cases (\%) } \quad \text { Male controls (\%) } p \text { value }^{\mathrm{a}} \text { Adjusted }
$$
$p$ value $^{\mathrm{b}} \quad p$ value $^{\mathrm{b}}$

\begin{tabular}{lllllllll}
\hline Type 1 & & & & & & \\
Hip & $54.5 / 45.5 / 0$ & $54.5 / 45.5 / 0$ & 1.00 & 0.96 & $47.8 / 43.5 / 8.7$ & $78.3 / 21.7 / 0$ & 0.014 & 0.048 \\
Femoral neck & $45.5 / 54.5 / 0$ & $63.6 / 27.3 / 9.1$ & 0.62 & 0.51 & $21.7 / 34.8 / 43.5$ & $39.1 / 52.2 / 8.7$ & 0.025 & 0.054 \\
Spine & $81.8 / 18.2 / 0$ & $70.0 / 30.0 / 0$ & 0.48 & 0.59 & $54.5 / 27.3 / 18.2$ & $73.9 / 26.1 / 0$ & 0.12 & 0.19 \\
Forearm & $81.8 / 18.2 / 0$ & $72.7 / 27.3 / 0$ & 0.56 & 0.63 & $52.2 / 34.8 / 13.0$ & $60.9 / 34.8 / 4.3$ & 0.36 & 0.57 \\
Type 2 & & & & & & & & \\
Hip & $76.7 / 20.9 / 2.3$ & $44.2 / 48.8 / 7.0$ & $<0.001$ & 0.15 & $75.5 / 23.6 / 0.9$ & $66.0 / 27.4 / 6.6$ & 0.11 & 0.78 \\
Femoral neck & $54.7 / 31.4 / 14.0$ & $18.6 / 55.8 / 25.6$ & $<0.001$ & 0.046 & $37.7 / 51.9 / 10.4$ & $25.5 / 51.9 / 22.6$ & 0.015 & 0.30 \\
Spine & $70.9 / 22.1 / 7.0$ & $52.3 / 36.0 / 11.6$ & 0.016 & 0.81 & $82.4 / 13.9 / 3.7$ & $72.9 / 21.5 / 5.6$ & 0.11 & 0.62 \\
Forearm & $69.0 / 22.6 / 8.3$ & $32.1 / 47.6 / 20.2$ & $<0.001$ & 0.002 & $56.5 / 38.0 / 5.6$ & $53.7 / 37.0 / 9.3$ & 0.52 & 0.92 \\
\hline
\end{tabular}

${ }^{\text {a }}$ Score statistic for type 3 Generalised Estimating Equation analysis

${ }^{\mathrm{b}}$ Adjusted for age and BMI

at the spine, after adjusting for age and BMI. In the men, adjusting for age and BMI removed the effect of type 2 diabetes on BMD at the hip sites.

A higher proportion of type 2 diabetic men had normal BMD than controls but this was significant at the femoral neck only, and when adjusted for age and BMI was no longer apparent. Similarly for women, a significantly higher proportion of diabetic women had normal BMD at each site and a lower proportion with osteopenia and osteoporosis, compared with controls, and this persisted even after adjusting for age and BMI.
Demographic, physical and biochemical associates of BMD

In the men with type 1 diabetes, BMD was significantly lower at hip, spine and femoral neck $(p \leq 0.013)$, but not at forearm $(p=0.20)$, compared with men with type 2 diabetes. After adjustment for age and BMI, these differences remained significant for the hip and femoral neck $(p \leq 0.033)$, but not for the spine $(p=0.24)$. The women with type 1 diabetes had similar BMD to those with type 2 diabetes at the spine, forearm and femoral neck $(p \geq 0.46)$,

Table 4 Univariate analyses of bone turnover markers and related hormones in 70 type 1 and type 2 diabetic men and women

\begin{tabular}{|c|c|c|c|c|c|c|}
\hline & \multicolumn{3}{|l|}{ Females } & \multicolumn{3}{|l|}{ Males } \\
\hline & Type $1(n=10)$ & Type $2(n=14)$ & $p$ value & Type $1(n=23)$ & Type $2(n=23)$ & $p$ value \\
\hline Age (years) & $43.4 \pm 10.2$ & $67.2 \pm 11.1$ & $<0.001$ & $54.4 \pm 14.3$ & $64.3 \pm 9.4$ & 0.008 \\
\hline BMI $\left(\mathrm{kg} / \mathrm{m}^{2}\right)$ & $25.4 \pm 4.9$ & $30.7 \pm 5.5$ & 0.024 & $25.9 \pm 3.2$ & $29.2 \pm 3.4$ & 0.003 \\
\hline Serum albumin $(\mathrm{g} / \mathrm{l})$ & $43.5[42.0-44.5]$ & $44.5[41.3-47.0]$ & 0.67 & $44.0[42.0-46.0]$ & $45.0[44.0-47.0]$ & 0.14 \\
\hline Serum calcium $(\mathrm{mmol} / \mathrm{l})$ & $2.4[2.0-2.5]$ & $2.4[1.3-2.5]$ & 0.94 & $2.4[2.2-2.5]$ & $2.4[2.1-2.6]$ & 0.35 \\
\hline Serum phosphate $(\mathrm{mmol} / \mathrm{l})$ & $1.2 \pm 0.2$ & $1.2 \pm 0.2$ & 0.99 & $1.2 \pm 0.2$ & $1.1 \pm 0.1$ & 0.31 \\
\hline Serum alkaline phosphatase (U/l) & $77.0 \pm 39.4$ & $80.8 \pm 36.1$ & 0.82 & $69.7 \pm 30.5$ & $61.2 \pm 29.2$ & 0.39 \\
\hline Serum osteocalcin (ng/ml) & $20.1(14.4-28.2)$ & $18.4(12.7-26.6)$ & 0.55 & $25.9(13.3-50.6)$ & $12.1(6.9-21.4)$ & 0.001 \\
\hline $\begin{array}{l}\text { Serum carboxyl-terminal type I } \\
\text { collagen telopeptide (ng/l) }\end{array}$ & $0.15(0.08-0.28)$ & $0.17(0.07-0.40)$ & 0.71 & $0.25(0.10-0.64)$ & $0.21(0.07-0.56)$ & 0.51 \\
\hline Serum vitamin D (nmol/1) & $61.7 \pm 15.9$ & $71.1 \pm 18.9$ & 0.23 & $64.9 \pm 17.4$ & $72.3 \pm 27.7$ & 0.33 \\
\hline Serum parathyroid hormone (pmol/l) & $3.8(2.3-6.4)$ & $4.0(2.4-6.7)$ & 0.78 & $5.6(2.7-11.9)$ & $3.7(2.2-6.1)$ & 0.029 \\
\hline $\begin{array}{l}\text { Serum sex hormone-binding } \\
\text { globulin }(\mathrm{nmol} / \mathrm{l})\end{array}$ & $55.3 \pm 16.6$ & $32.2 \pm 11.2$ & 0.001 & $41.8 \pm 17.9$ & $28.4 \pm 15.7$ & 0.021 \\
\hline Total serum testosterone $(\mathrm{nmol} / \mathrm{l})$ & $1.4[1.2-1.5]$ & $1.1[0.7-1.5]$ & 0.23 & $19.0[13.6-24.7]$ & $14.2[10.8-16.7]$ & 0.001 \\
\hline Serum free testosterone $(\mathrm{nmol} / \mathrm{l})^{a}$ & $0.02^{\mathrm{b}}$ & $0.02 \pm 0.02$ & 0.49 & $0.38 \pm 0.09$ & $0.33 \pm 0.11$ & 0.16 \\
\hline Serum oestradiol (pmol/1) & $212(52-859)$ & $18(6-54)$ & $<0.001$ & $86(49-151)$ & $61(23-163)$ & 0.15 \\
\hline Serum luteinising hormone (U/l) & $4.7(1.4-15.8)$ & $12.7(5.0-31.9)$ & 0.042 & $4.6(2.3-9.1)$ & $4.3(2.0-9.4)$ & 0.80 \\
\hline Serum follicle-stimulating hormone (U/1) & $6.6(1.7-26.6)$ & $29.6(9.2-95.4)$ & 0.013 & $6.3(2.5-15.6)$ & $8.0(3.4-18.9)$ & 0.43 \\
\hline
\end{tabular}

Values are mean $\pm \mathrm{SD}$, geometric mean (SD range) or median [interquartile range]

${ }^{a}$ Serum free testosterone was calculated for patients with E2 $<150 \mathrm{pmol} / 1$

${ }^{\mathrm{b}} n=2$ with same value 
Table 5 Independent associates of forearm, spine, hip and femoral neck BMD assessed by multiple linear regression analysis

\begin{tabular}{|c|c|c|c|c|c|c|c|c|c|}
\hline & \multicolumn{3}{|c|}{ Females $(n=24)$} & \multicolumn{3}{|c|}{ Males $(n=46)$} & \multicolumn{3}{|c|}{ All patients $(n=70)$} \\
\hline & $B^{\mathrm{a}}$ & $p$ value & Adjusted $R^{2}$ & $B^{\mathrm{a}}$ & $p$ value & Adjusted $R^{2}$ & $B$ & $p$ value & Adjusted $R^{2}$ \\
\hline \multicolumn{10}{|l|}{ Forearm } \\
\hline BMI & - & - & - & 0.008 & 0.003 & 0.168 & 0.005 & 0.001 & 0.056 \\
\hline $\ln (\mathrm{E} 2)$ & 0.018 & 0.009 & 0.252 & - & - & - & 0.013 & 0.020 & 0.035 \\
\hline $\ln (\mathrm{CTx})$ & - & - & - & - & - & - & -0.021 & 0.010 & 0.061 \\
\hline Male sex & - & - & - & - & - & - & 0.115 & $<0.001$ & 0.458 \\
\hline All & - & - & 0.252 & - & - & 0.168 & - & - & 0.610 \\
\hline \multicolumn{10}{|l|}{ Spine } \\
\hline BMI & - & - & - & 0.025 & 0.001 & 0.171 & 0.013 & 0.003 & 0.106 \\
\hline $\ln (\mathrm{E} 2)$ & 0.036 & 0.013 & 0.222 & 0.068 & 0.039 & 0.067 & 0.052 & 0.002 & 0.081 \\
\hline All & - & - & 0.222 & - & - & 0.238 & - & - & 0.19 \\
\hline \multicolumn{10}{|l|}{ Hip } \\
\hline BMI & 0.018 & $<0.001$ & 0.447 & 0.024 & $<0.001$ & 0.187 & 0.023 & $<0.001$ & 0.288 \\
\hline $\ln (\mathrm{CTx})$ & - & - & - & -0.073 & 0.004 & 0.268 & -0.061 & 0.002 & 0.118 \\
\hline $\ln (\mathrm{E} 2)$ & 0.027 & 0.039 & 0.090 & 0.057 & 0.049 & 0.050 & 0.031 & 0.026 & 0.038 \\
\hline Male sex & - & - & - & - & - & - & 0.147 & $<0.001$ & 0.106 \\
\hline All & - & - & 0.537 & - & - & 0.505 & - & - & 0.550 \\
\hline \multicolumn{10}{|c|}{ Femoral neck } \\
\hline Age & -0.004 & 0.004 & 0.181 & - & - & - & - & - & - \\
\hline BMI & 0.016 & $<0.001$ & 0.271 & 0.016 & 0.004 & 0.148 & 0.017 & $<0.001$ & 0.246 \\
\hline $\ln (\mathrm{CTx})$ & -0.062 & 0.029 & 0.113 & -0.074 & 0.001 & 0.282 & -0.057 & 0.001 & 0.100 \\
\hline $\ln (\mathrm{E} 2)$ & - & - & - & - & - & - & 0.028 & 0.015 & 0.053 \\
\hline Male sex & - & - & - & - & - & - & 0.088 & 0.003 & 0.096 \\
\hline All & - & - & 0.565 & - & - & 0.430 & - & - & 0.495 \\
\hline
\end{tabular}

${ }^{\mathrm{a}} B$ unstandardised regression coefficient

CTx Carboxyl-terminal type I collagen telopeptide; E2 oestradiol

but significantly lower BMD at the hip $(p<0.015)$. After adjusting for age and BMI, BMD at both hip and femoral neck were significantly lower for the type 1 diabetic women $(p \leq 0.047)$. However, when menopausal status and HRT were also included, only the difference in BMD at the hip remained significant $(p=0.023)$.

For all the type 2 patients, and after adjusting for age, sex and BMI using multiple linear regression, BMD at the hip and femoral neck were negatively associated with $\mathrm{HbA}_{1 \mathrm{c}}$ $(p \leq 0.003)$ and $\ln$ (serum triglycerides) $(p \leq 0.009)$. BMD at the spine was associated with diastolic $\mathrm{BP}(p \leq 0.001)$, while forearm BMD was not associated with metabolic control, $\mathrm{BP}$ or diabetes duration. BMD at the femoral neck was significantly associated with neuropathy $(p=0.022)$ but not CHD, cerebrovascular disease, retinopathy or nephropathy. BMD at the other sites was not associated with these diabetic complications. There were insufficient numbers of type 1 patients to undertake similar analyses.

\section{Biochemical markers of bone metabolism}

Table 4 summarises biochemical data for the type 1 and a sample of the type 2 diabetic patients matched by sex and serum creatinine from whom sera were available $(n=70)$. Men with type 1 diabetes had higher osteocalcin $(p=0.001)$, PTH $(p=0.029)$, SHBG $(p=0.021)$ and total testosterone
( $p=0.001)$ than type 2 men, while in women with type 1 diabetes both SHBG $(p=0.001)$ and E2 $(p<0.001)$ were higher, and LH $(p=0.042)$ and FSH $(p=0.013)$ were lower, than in those with type 2 diabetes. No differences in albumin, ALP, calcium and CTx concentrations were found between type 1 and type 2 patients in the case of either men or women.

In multiple linear regression analysis of all 46 men with BMD as the dependent variable (Table 5), BMI was an independent predictor of BMD at each site. $\ln (\mathrm{E} 2)$ was significantly associated with BMD at spine and hip but explained less of the variance in BMD than BMI. $\ln (\mathrm{CTx})$ was associated with BMD at hip and femoral neck and accounted for a greater proportion of the variance in BMD than BMI. In the 24 women, $\ln (\mathrm{E} 2)$ was independently associated with forearm, spine and hip BMD. BMI was strongly associated with hip BMD and, together with $\ln (\mathrm{E} 2)$, accounted for $54 \%$ of the total variance in BMD at this site. BMI, $\ln (\mathrm{CTx})$ and age were independently associated with femoral neck BMD, accounting for $57 \%$ of the total variance.

When data from all 70 patients were pooled, BMI, $\ln (\mathrm{E} 2), \ln (\mathrm{CTx})$ and male sex were consistently and independently associated with BMD at forearm, hip and femoral neck. The inclusion of these four variables explained 61,55 and $50 \%$, respectively, of the total variance in BMD at these sites, with BMI accounting 
for no more than a half of this. Spine BMD was independently associated with BMI and $\ln (\mathrm{E} 2)$ only. Other variables considered for entry into the models included $\ln (\mathrm{PTH})$, insulin use, vitamin $\mathrm{D}$, calcium and serum creatinine, but these were not independently associated with BMD. After adjusting for the variables in the models presented in Table 5, metabolic control, $\mathrm{BP}$ and vascular complications were not independently associated with BMD by site or sex, except for BMD at the spine in females. Total serum cholesterol added significantly to this model (regression coefficient, $B=0.11, p=0.005$, adjusted $R^{2}=0.45$ ). None of the 70 patients were taking HRT at the time of densitometry and few were smokers, regular alcohol consumers or (in the case of those with type 2 diabetes) insulin-treated, variables that did not prove significant in the various regression models assessed.

\section{Fracture data}

Analysis of fractures was limited by their small number in the Linked Database. Only two type 1 patients $(5.9 \%)$ had been hospitalised for fracture between 1993 and 2003; one suffered two fractures (forearm and femur) while the second suffered a fractured metacarpal bone following a hypoglycaemic episode. Similarly, only $12(6.3 \%)$ of the 189 type 2 patients with linked data were admitted with a fracture, seven before BMD was measured. For selfreported fracture, 55 patients had at least one before recruitment to the present sub-study. There was no correlation between fracture site and osteoporosis from self-reported or Linked Database fractures (data not shown).

\section{Discussion}

The present case-control analyses suggest that the patient's sex has an important effect on BMD in diabetes. Our men with type 1 diabetes had reduced BMD at hip and femoral neck compared with controls, which was independent of age and BMI. There was no such difference in the case of the women with type 1 diabetes in our cohort. Although ageand BMI-adjusted BMD in our type 2 males was similar to that of matched controls, the type 2 women had increased BMD at hip, femoral neck and forearm. In multivariate regression models involving a subset of patients, male sex remained a significant predictor of BMD together with CTx and serum E2. Up to two-thirds of the total variance in BMD at femoral neck, hip and forearm could be explained by these three variables and BMI.

Although we did not show any association between BMD and glycaemic control, disease duration, serum lipids, blood pressure or complications in our type 1 patients, diabetic status alone was an important predictor of lower BMD in the males. This finding is in contrast to two previous studies that showed reduced BMD at proximal femur in both men and women [12, 31]. Direct compar- isons are, however, difficult, since, in one of these studies, the type 1 patients were significantly older than those in our community-based cohort and had been diabetic for $>30$ years, while in the other, type 1 patients were not matched with the control subjects [31]. Our data parallel the results of a longitudinal study that showed greater reductions in spine and total BMD in type 1 men than women over a two-year period [32]. However, the strongest associations between male sex and BMD in our sample were for sites other than the spine. An alternative explanation for the non-significant effects in type 1 women is a type II error, with the small sample size associated with $\leq 31 \%$ power to detect a one-sided significant difference with the observed BMD values in this group.

In type 2 diabetes, both men and women had higher BMD than matched controls in the present study. However, in the type 2 diabetic men, the difference disappeared after adjusting for age and BMI. By contrast, diabetes was associated with higher BMD at three of the four sites measured in the women, independently of age and BMI. Our findings are in agreement with several previous studies that also demonstrated increased BMD at lumbar spine, femoral neck and forearm in type 2 diabetic patients [33], a relationship that was stronger for women [31,34] even after adjusting for adiposity and lifestyle variables [34, 35]. The fact that diabetes status was a significant predictor of BMD in women independently of BMI may be partly explained by the anabolic effect that insulin has on bone tissue. Since type 2 diabetes is preceded by a period of insulin resistance, hyperinsulinaemia may confer a protective effect on BMD, either directly through elevated fasting insulin as demonstrated in diabetic and non-diabetic elderly men and women [36, 37] or indirectly through BMI [35]. Of potential relevance to the present findings is that there is a rise in serum insulin with advancing age in women from the 5 th to the 8 th decade and a concomitant decline in men, consistent with the greater BMD in our women with type 2 diabetes compared with matched controls.

We measured bone turnover markers and sex hormone levels in a subgroup of our patients to determine whether these variables help to explain the sex- and diabetesspecific differences in BMD. A number of recent studies outside the context of diabetes have reported the importance of E2 in maintaining BMD [18, 20, 38], and that low serum E2 rather than testosterone concentrations are a risk factor for osteoporosis in men [20] and women [18]. Our data provide evidence that this also applies in diabetes, with $\ln (\mathrm{E} 2)$ a strong independent predictor of BMD in women (forearm, spine and hip), men (spine and hip) and in all subjects combined (all sites). Diabetic men are reported to have higher serum E2 concentrations than control males [39], and these elevated levels can be further increased with improved glycaemic control [40]. In the total sample of 194 type 2 patients, $\mathrm{HbA}_{1 \mathrm{c}}$ was independently associated with BMD at hip and femoral neck and it may be that this relationship is due, in part, to higher serum E2 levels in the better-controlled patients. We did not find 
that serum total testosterone, SHBG or free $\mathrm{T}$ predicted BMD at any site.

The bone resorption marker CTx was a significant independent predictor of BMD in women (femoral neck), men (hip and femoral neck) and in all subjects (forearm, hip and femoral neck), with a consistent inverse association between serum levels and BMD. We did not show any association between BMD and serum calcium, phosphate, ALP, osteocalcin and vitamin D in either type 1 or type 2 diabetes, a result that is consistent with those of other studies [31, 32]. These data suggest that diabetesassociated reductions in BMD relate primarily to increased bone resorption, and that bisphosphonate therapy could be valuable in the treatment of diabetic patients with, or at high risk of, accelerated skeletal calcium loss.

A valid assessment of the impact of BMD on fractures severe enough to justify hospitalisation was not possible since there were few such events in our patients. Although there were greater numbers of self-reported fractures, there was no association with BMD-defined osteoporosis. In larger-scale studies, type 1 diabetes increases the risk of fracture, especially in those with microvascular complications $[2,14,41]$ and in females [42, 43]. In type 2 diabetes, both lower [44] and higher [1] rates of fracture have been reported, with disease duration and use of insulin increasing the risk [42]. A recent study has reported that type 2 diabetes was a risk factor for hip, proximal humerus and foot fracture despite the presence of high BMD [45]. This would suggest that the high BMD seen in our type 2 diabetic patients may not parallel bone quality or strength.

The present study had limitations. Our sample size was small, particularly in the case of type 1 patients. As a result, the present data should be interpreted as preliminary. Nevertheless, we had more comprehensive data on each patient than in most previous studies of osteoporosis in diabetes. Our data were cross-sectional and therefore it is not possible to impute causality based on our findings. Further large-scale prospective studies of diabetes-associated changes in BMD and its determinants would address these limitations.

The present results provide some evidence that men with type 1 diabetes are significantly disadvantaged with respect to hip BMD and are at greatest risk of developing osteoporosis. Indeed, $>50 \%$ of our type 1 males had osteopenia or osteoporosis. By contrast, women with type 2 diabetes may have protection against fractures as a result of increased BMD at several sites. E2 and CTx appear to be strong predictors of BMD in diabetes. However, the balance of bone preservation and resorption is determined by additional variables that include male sex and BMI. We were not able to assess the relationship between BMD, bone fracture and diabetes status in our cohort, but continued acquisition of fracture data through the Linked Database may provide useful future outcome data from the present cohort.
Acknowledgements We are grateful to the Fremantle Diabetes Study and Fremantle Hospital and Health Service staff for patient recruitment, data collection and assistance with assays, as well as to R. Price from Sir Charles Gairdner Hospital for providing control subjects from the local healthy population. The present study was supported by a grant from the Fremantle Hospital Research Foundation. The Raine Foundation, University of Western Australia funded the Fremantle Diabetes Study.

\section{References}

1. Meyer HE, Tverdal A, Falch JA (1993) Risk factors for hip fracture in middle-aged Norwegian women and men. Am J Epidemiol 137:1203-1211

2. Ivers RQ, Cumming RG, Mitchell P, Peduto AJ (2001) Diabetes and risk of fracture: the Blue Mountains Eye study. Diabetes Care 24:1198-1203

3. Vogt MT, Cauley JA, Tomaino MM, Stone K, Williams JR, Herndon JH (2002) Distal radius fractures in older women: a 10 -year follow-up study of descriptive characteristics and risk factors. The study of osteoporotic fractures. J Am Geriatr Soc 50:97-103

4. Soejima K, Landing BH (1986) Osteoporosis in juvenile-onset diabetes mellitus: morphometric and comparative studies. Pediatr Pathol 6:289-299

5. Lopez-Ibarra PJ, Pastor MM, Escobar-Jimenez F et al (2001) Bone mineral density at time of clinical diagnosis of adult-onset type 1 diabetes mellitus. Endocr Pract 7:346-351

6. Lettgen B, Hauffa B, Mohlmann C, Jeken C, Reiners C (1995) Bone mineral density in children and adolescents with juvenile diabetes: selective measurement of bone mineral density of trabecular and cortical bone using peripheral quantitative computed tomography. Horm Res 43:173-175

7. Valerio G, del Puente A, Esposito-del Puente A, Buono P, Mozzillo E, Franzese A (2002) The lumbar bone mineral density is affected by long-term poor metabolic control in adolescents with type 1 diabetes mellitus. Horm Res 58:266-272

8. Heap J, Murray MA, Miller SC, Jalili T, Moyer-Mileur LJ (2004) Alterations in bone characteristics associated with glycemic control in adolescents with type 1 diabetes mellitus. J Pediatr 144:56-62

9. Saggese G, Bertelloni S, Baroncelli GI, Federico G, Calisti L, Fusaro C (1989) Bone demineralization and impaired mineral metabolism in insulin-dependent diabetes mellitus. A possible role of magnesium deficiency. Helv Paediatr Acta 43:405-414

10. Hanley DA, Brown JP, Tenenhouse A et al (2003) Associations among disease conditions, bone mineral density, and prevalent vertebral deformities in men and women 50 years of age and older: cross-sectional results from the Canadian Multicentre Osteoporosis study. J Bone Miner Res 18:784-790

11. Bauer DC, Browner WS, Cauley JA et al (1993) Factors associated with appendicular bone mass in older women. The Study of Osteoporotic Fractures Research Group. Ann Intern Med 118:657-665

12. Tuominen JT, Impivaara O, Puukka P, Ronnemaa T (1999) Bone mineral density in patients with type 1 and type 2 diabetes. Diabetes Care 22:1196-1200

13. Hampson G, Evans C, Petitt RJ et al (1998) Bone mineral density, collagen type 1 alpha 1 genotypes and bone turnover in premenopausal women with diabetes mellitus. Diabetologia 41:1314-1320

14. Piepkorn B, Kann P, Forst T, Andreas J, Pfutzner A, Beyer $\mathrm{J}$ (1997) Bone mineral density and bone metabolism in diabetes mellitus. Horm Metab Res 29:584-591

15. Mathiassen B, Nielsen S, Ditzel J, Rodbro P (1990) Long-term bone loss in insulin-dependent diabetes mellitus. J Intern Med 227:325-327 
16. Olmos JM, Perez-Castrillon JL, Garcia MT, Garrido JC, Amado JA, Gonzalez-Macias J (1994) Bone densitometry and biochemical bone remodeling markers in type 1 diabetes mellitus. Bone Miner 26:1-8

17. Levin ME, Boisseau VC, Avioli LV (1976) Effects of diabetes mellitus on bone mass in juvenile and adult-onset diabetes. $\mathrm{N}$ Engl J Med 294:241-245

18. Khosla S, Melton LJ 3rd, Atkinson EJ, O'Fallon WM, Klee GG, Riggs BL (1998) Relationship of serum sex steroid levels and bone turnover markers with bone mineral density in men and women: a key role for bioavailable estrogen. J Clin Endocrinol Metab 83:2266-2274

19. Amin S, Zhang Y, Sawin CT et al (2000) Association of hypogonadism and estradiol levels with bone mineral density in elderly men from the Framingham study. Ann Intern Med 133:951-963

20. Szulc P, Munoz F, Claustrat B et al (2001) Bioavailable estradiol may be an important determinant of osteoporosis in men: the MINOS study. J Clin Endocrinol Metab 86:192-199

21. Davis TM, Zimmet P, Davis WA, Bruce DG, Fida S, Mackay IR (2000) Autoantibodies to glutamic acid decarboxylase in diabetic patients from a multi-ethnic Australian community: the Fremantle Diabetes Study. Diabet Med 17:667-674

22. Price RI, Walters MJ, Retallack RW et al (2003) Impact of the analysis of a bone density reference range on determination of the T-score. J Clin Densitom 6:51-62

23. Gillett M, Davis WA, Jackson D, Bruce DG, Davis TM (2003) Prospective evaluation of carotid bruit as a predictor of first stroke in type 2 diabetes: the Fremantle Diabetes Study. Stroke $34: 2145-2151$

24. Feldman EL, Stevens MJ, Thomas PK, Brown MB, Canal N, Greene DA (1994) A practical two-step quantitative clinical and electrophysiological assessment for the diagnosis and staging of diabetic neuropathy. Diabetes Care 17:1281-1289

25. Davis TM, Fortun P, Mulder J, Davis WA, Bruce DG (2004) Silent myocardial infarction and its prognosis in a communitybased cohort of type 2 diabetic patients: the Fremantle Diabetes Study. Diabetologia 47:395-399

26. Genant HK, Cooper C, Poor G et al (1999) Interim report and recommendations of the World Health Organization Task-Force for Osteoporosis. Osteoporos Int 10:259-264

27. Henzell S, Dhaliwal S, Pontifex R et al (2000) Precision error of fan-beam dual X-ray absorptiometry scans at the spine, hip and forearm. J Clin Densitom 3:359-364

28. Vermeulen A, Verdonck L, Kaufman JM (1999) A critical evaluation of simple methods for the estimation of free testosterone in serum. J Clin Endocrinol Metab 84:3666-3672

29. Smeets-Goevaers CG, Lesusink GL, Papapoulos SE et al (1998) The prevalence of low bone mineral density in Dutch perimenopausal women: the Eindhoven perimenopausal osteoporosis study. Osteoporos Int 8:404-409

30. Kroger H, Tuppurainen M, Honkanen R, Alhava E, Saarikoski $\mathrm{S}$ (1994) Bone mineral density and risk factors for osteoporosis - a population-based study of 1600 perimenopausal women. Calcif Tissue Int 55:1-7
31. Buysschaert M, Cauwe F, Jamart J et al (1992) Proximal femur density in type 1 and 2 diabetic patients. Diabete Metab 18: 32-37

32. Miazgowski T, Czekalski S (1998) A 2-year follow-up study on bone mineral density and markers of bone turnover in patients with long-standing insulin-dependent diabetes mellitus. Osteoporos Int 8:399-403

33. Lunt M, Masaryk P, Scheidt-Nave C et al (2001) The effects of lifestyle, dietary dairy intake and diabetes on bone density and vertebral deformity prevalence: the EVOS study. Osteoporos Int 12:688-698

34. Barrett-Connor E, Holbrook TL (1992) Sex differences in osteoporosis in older adults with non-insulin-dependent diabetes mellitus. JAMA 268:3333-3337

35. Dennison EM, Syddall HE, Aihie Sayer A, Craighead S, Phillips DI, Cooper C (2004) Type 2 diabetes mellitus is associated with increased axial bone density in men and women from the Hertfordshire Cohort study: evidence for an indirect effect of insulin resistance? Diabetologia 47:1963-1968

36. Barrett-Connor E, Kritz-Silverstein D (1996) Does hyperinsulinemia preserve bone? Diabetes Care 19:1388-1392

37. Stolk RP, Van Daele PL, Pols HA et al (1996) Hyperinsulinemia and bone mineral density in an elderly population: the Rotterdam study. Bone 18:545-549

38. Rapuri PB, Gallagher JC, Haynatzki G (2004) Endogenous levels of serum estradiol and sex hormone binding globulin determine bone mineral density, bone remodeling, the rate of bone loss, and response to treatment with estrogen in elderly women. J Clin Endocrinol Metab 89:4954-4962

39. Small M, MacRury S, Beastall GH, MacCuish AC (1987) Oestradiol levels in diabetic men with and without a previous myocardial infarction. Q J Med 64:617-623

40. Christensen L, Hagen C, Henriksen JE, Haug E (1997) Elevated levels of sex hormones and sex hormone binding globulin in male patients with insulin dependent diabetes mellitus. Effect of improved blood glucose regulation. Dan Med Bull 44:547-550

41. Cundy TF, Edmonds ME, Watkins PJ (1985) Osteopenia and metatarsal fractures in diabetic neuropathy. Diabet Med 2:461-464

42. Nicodemus KK, Folsom AR (2001) Type 1 and type 2 diabetes and incident hip fractures in postmenopausal women. Diabetes Care 24:1192-1197

43. Forsen L, Meyer HE, Midthjell K, Edna TH (1999) Diabetes mellitus and the incidence of hip fracture: results from the Nord-Trondelag Health Survey. Diabetologia 42:920-925

44. Heath H 3rd, Melton LJ 3rd, Chu CP (1980) Diabetes mellitus and risk of skeletal fracture. N Engl J Med 303:567-570

45. Schwartz AV, Sellmeyer DE, Ensrud KE et al (2001) Older women with diabetes have an increased risk of fracture: a prospective study. J Clin Endocrinol Metab 86:32-38 